\title{
The Use of Event-Related Potentials and Machine Learning to Improve Diagnostic Testing and Prediction of Disease Progression in Parkinson's Disease
}

\author{
Robin VLIEGER ${ }^{\mathrm{a}, 1}$, Elena DASKALAKI ${ }^{\mathrm{a}}$, Deborah APTHORP ${ }^{\mathrm{a}, \mathrm{b}}$, \\ Christian J LUECK ${ }^{\mathrm{c}, \mathrm{d}}$ and Hanna SUOMINEN ${ }^{\mathrm{a}, \mathrm{e}, \mathrm{f}}$ \\ ${ }^{\text {a }}$ School of Computing, Australian National University, ANU, Australia \\ ${ }^{\mathrm{b}}$ School of Psychology, University of New England, Australia \\ ${ }^{c}$ ANU Medical School, ANU, Australia \\ ${ }^{\mathrm{d}}$ Department of Neurology, Canberra Hospital, Australia \\ ${ }^{\mathrm{e}}$ Data61, Commonwealth Scientific and Industrial Research Organisation, Australia \\ ${ }_{\mathrm{f}}$ Department of Computing, University of Turku, Finland
}

\begin{abstract}
Current tests of disease status in Parkinson's disease suffer from high variability, limiting their ability to determine disease severity and prognosis. Eventrelated potentials, in conjunction with machine learning, may provide a more objective assessment. In this study, we will use event-related potentials to develop machine learning models, aiming to provide an objective way to assess disease status and predict disease progression in Parkinson's disease.
\end{abstract}

Keywords. Diagnosis, event-related potentials, machine learning, Parkinson disease, disease status, prediction

\section{Introduction}

Current methods of evaluating disease severity in Parkinson's disease (PD) suffer from high variability, making it hard to determine disease status and predict disease progression accurately [1]. Machine learning (ML) allows analysis of multivariate and complex data in a manner that is more objective than most conventional methods and has been used in the prediction of disease progression in other degenerative diseases such as Alzheimer's Disease [2]. In the case of PD, event-related potentials (ERPs), being brain responses to sensory, cognitive, or motor events, have been shown to differ between people with the disease and controls [3] and, thus, might provide an objective assessment of disease severity. This study examines the contingent negative variation $(\mathrm{CNV})$, an electrical potential that develops in anticipation of a response to a target.

\footnotetext{
${ }^{1}$ Corresponding Author, Robin Vlieger, School of Computing, Australian National University, Hanna Neumann Building, 145 Science Road, Acton, ACT 2601, Australia; E-mail: robin.vlieger@anu.edu.au.
} 


\section{Approach}

The study was performed at the Canberra Hospital and ethics approval was obtained from the ACT Health Human Research Ethics Committee under protocol number ETH.4.16.060. Electroencephalography (EEG) recordings were made using a 64-channel ActiveTwo BioSemi system. Participants were seated in a chair in front of a projector screen and will respond via a RESPONSEpixx 5-button response box.

A blue square was presented in the centre of a white screen, and $2.5 \mathrm{sec}$ later, an $\mathrm{X}$ or a $\mathrm{Y}$ was presented to either the right or the left of the cue. The participant responded to the $\mathrm{X}$ by pressing the button that corresponded to the side of appearance of the $\mathrm{X}$ while withholding any response to the Y. An X appeared in $80 \%$ of the trials and the experiment included 90 trials.

Data from 21 people with PD and 16 controls were processed in MATLAB R2019a and EEGlab. The CNV was quantified by subtracting the mean amplitude in the 2000 $1500 \mathrm{~ms}$ interval from the $500-0 \mathrm{~ms}$ interval pre-stimulus. The resultant features were used as input to a random forest algorithm and the electrodes that were most prevalent after running the algorithm 100 times selected as input to a support vector machine (SVM) for ML-based classification. Leave-one-out cross-validation (LOOCV) was used for classification evaluation.

\section{Results}

Because the early $\mathrm{CNV}$ differs between people with other neurodegenerative diseases and controls [4], we expected similar differences to be present between people with PD and controls. The electrodes selected by the random forest algorithm corresponded with the literature and the combination of the SVM with LOOCV produced an accuracy of $72.97 \%$, with sensitivity of $71.43 \%$, specificity of $75 \%$, and precision of $78.95 \%$.

\section{Conclusions}

The results show the CNV has promise as a marker of disease status in PD, while leaving room for improvement. The $\mathrm{CNV}$ is a slow-building potential and mean amplitudes may not be the best to capture subtle changes. EEG data are noisy and different electrodes often pick up on the same signal to varying degrees. Dimensionality and noise reduction techniques, such as Principal Component Analysis, might be able to improve classification accuracy. Therefore, different quantification methods, as well as dimensionality and noise reduction techniques are avenues for future work.

\section{Acknowledgements}

This research was funded by and has been delivered in partnership with Our Health in Our Hands (OHIOH), a strategic initiative of the ANU, which aims to transform health care by developing new personalized health technologies and solutions in collaboration with patients, clinicians, and health-care providers. We gratefully acknowledge the funding from the ANU School of Computing for the first author's PhD studies. 


\section{References}

[1] Evers LJ, Krijthe JH, Meinders MJ, Bloem BR, Heskes TM. (2019). Measuring Parkinson's disease over time: The real-world within-subject reliability of the MDS-UPDRS. Movement Disorders, 34(10), 14801487.

[2] Moradi E, Pepe A, Gaser C, Huttunen H, Tohka J, A.s.D.N. Initiative (2015), Machine learning framework for early MRI-based Alzheimer's conversion prediction in MCI subjects. Neuroimage, 104, 398-412.

[3] Seer C, Lange F, Georgiev D, Jahanshahi M, Kopp B. (2016). Event-related potentials and cognition in Parkinson's disease: An integrative review. Neuroscience \& Biobehavioral Reviews, 71, 691-714.

[4] Turner LM, Croft RJ, Churchyard A, Looi JC, Apthorp D, Georgiou-Karistianis N. Abnormal electrophysiological motor responses in Huntington's disease: Evidence of premanifest compensation. (2015), PloS One 10, e0138563. 